\title{
Non-extensive Trends in the Size Distribution of Coding and Non-coding DNA Sequences in the Human Genome
}

\author{
Th. Oikonomou ${ }^{1,2 *}$ and A. Provata ${ }^{1} \dagger$ \\ ${ }^{1}$ Institute of Physical Chemistry, \\ National Center for Scientific Research "Demokritos" \\ 15310, Athens, Greece \\ 2 School of Medicine, Department of Biological Chemistry \\ University of Athens, Goudi, 11527, Athens, Greece
}

November 13, 2018

\begin{abstract}
We study the primary DNA structure of four of the most completely sequenced human chromosomes (including chromosome 19 which is the most dense in coding), using Non-extensive Statistics. We show that the exponents governing the decay of the coding size distributions vary between $5.2 \leq r \leq 5.7$ for the short scales and $1.45 \leq q \leq 1.50$ for the large scales. On the contrary, the exponents governing the decay of the non-coding size distributions in these four chromosomes, take the values $2.4 \leq r \leq 3.2$ for the short scales and $1.50 \leq q \leq 1.72$ for the large scales. This quantitative difference, in particular in the tail exponent $q$, indicates that the non-coding (coding) size distributions have long (short) range correlations. This non-trivial difference in the DNA statistics is attributed to the non-conservative (conservative) evolution dynamics acting on the non-coding (coding) DNA sequences.
\end{abstract}

PACS Numbers: 89.75.-k (Systems Obeying Scaling Laws); 87.17.Gg (DNA, RNA); 05.65.+b (Self-organised Systems).

\footnotetext{
${ }^{*}$ E-mail: thoikonomou@chem.demokritos.gr

${ }^{\dagger}$ Corresponding author, e-mail:aprovata@limnos.chem.demokritos.gr
} 
KEY WORDS: Non-extensive Statistics; Coding/non-coding DNA Sequences; Power law distributions; Long range correlations.

\section{Introduction}

During recent years numerous studies on the statistics of genomic sequences have demonstrated various degrees of complexity in the primary structure of DNA. In particular, Peng et al. in 1992 demonstrated the existence of long range correlations using the "DNA walk" model [1]. Similar conclusions were reached by Li et al. [2] and Voss [3] using the $1 / f$ spectrum and later by studies on the size distribution of Purine (Adenine, Guanine) and Pyrimidine (Thymine, Cytocine) clusters in coding and non-coding regions of different organisms 4, 5]. Other studies manifested long range correlations and power laws in the primary structure of DNA using a variety of statistical methods ranging from wavelets to linguistic approaches [6, 7, 8, 9, 10, 11.

In recent studies, one of the present authors (AP) and coworkers have shown that the long range distributions of Pyrine and Pyrimidine clusters in the non-coding regions of higher eucaryotes are related to similar long range distributions present at a higher level of genomic organisation: the level of coding and non-coding alternating regions [12, 13, 14].

Non-extensive Statistical Mechanics is particularly fitted to describe complex structures which present long range correlations, power laws and fractality [15]. In particular, non-extensive statistics have been used to describe successfully complex spatiotemporal structures in diverse fields such as high energy physics [16], turbulence [17, biological systems [18, anomalous diffusion [19, classical and quantum chaos [20, interacting particle systems [21] and reactive dynamics [22].

Classical Statistical Mechanics uses the Boltzmann Gibbs (BG) Entropy, $S_{B G}$, defined as:

$$
S_{B G}=-\sum_{i=1}^{W} p_{i} \ln p_{i}
$$

to describe the properties of systems at equilibrium. In Eq. 1] $p_{i}$ denotes the probabilities of the $i-t h$ microscopic state and the average runs over the total number of states $W$. This BG entropic form can not successfully describe systems in which self-organisation, long range features and scaling are observed. As a generalisation of Eq. 1] Tsallis and coworkers 223] have 
introduced the non-extensive entropy, defined as:

$$
S_{q}=\frac{1-\sum_{i=1}^{W} p_{i}^{q}}{q-1}, \text { for } q \neq 1
$$

where $q$ is the non-extensivity exponent. Note that for $q=1$ the classical BG statistics (Eq. 1) is recovered and thus departure of the exponent $q$ from the value 1 signals departure from BG statistics.

In relation to non-extensive statistics, long range decay maybe obtained by a non-linear dynamical process expressed by [24]:

$$
\frac{d \xi}{d s}=-\kappa_{q} \xi^{q}, \quad \text { for } \quad\left(\kappa_{q} \geq 0, q \neq 1\right)
$$

In particular, for $q>1$ long range decay is manifested, while for $q=1$ the well known exponential decay is obtained. The solution of Eq. 3] is:

$$
\begin{aligned}
\xi(s) & =\left[1-(1-q) \kappa_{q}(s-1)\right]^{1 /(1-q)}, \quad \text { for } \quad\left(\kappa_{q} \geq 0, q>1\right) \\
& =\exp \left(-\kappa_{1}(s-1)\right), \text { for } \quad\left(\kappa_{1} \geq 0, q=1\right)
\end{aligned}
$$

with initial condition $\xi(1)=1$. Thus for $q>1$ a long range law (power law decay) is obtained, while for $q=1$ a short range law (exponential decay) emerges.

For phenomena where two or more dynamical mechanisms act in the system producing different decay laws in different length (and/or time) scales, a further phenomenological generalisation of Eq. 3 maybe introduced by addition of terms carrying different powers [24]. The simplest one carries only one additional term and is:

$$
\frac{d \xi}{d s}=-\kappa_{q} \xi^{q}-\left(\lambda_{r}-\kappa_{q}\right) \xi^{r}, \quad \text { for } \quad(q \leq r)
$$

Note that Eq. 3] is recovered for $\kappa_{q}=\lambda_{r}(\forall r)$. The solution of Eq. [5 can not be written in a simple form but it may be shown that it consists of two distinct power law regions, one governed by the exponent $q$ and one by the exponent $r$ [24].

In Fig. 1 we present the size distribution of coding and non-coding DNA sequences in chromosome 16. To avoid local fluctuations running averages are considered over 15 Base Pairs (bps). For clarity only the first 1000 points are shown. The maximum size of coding regions is of the order of 7000-8000 bps (reaches 20000bps for chromosome 19) while the maximum sizes of the 


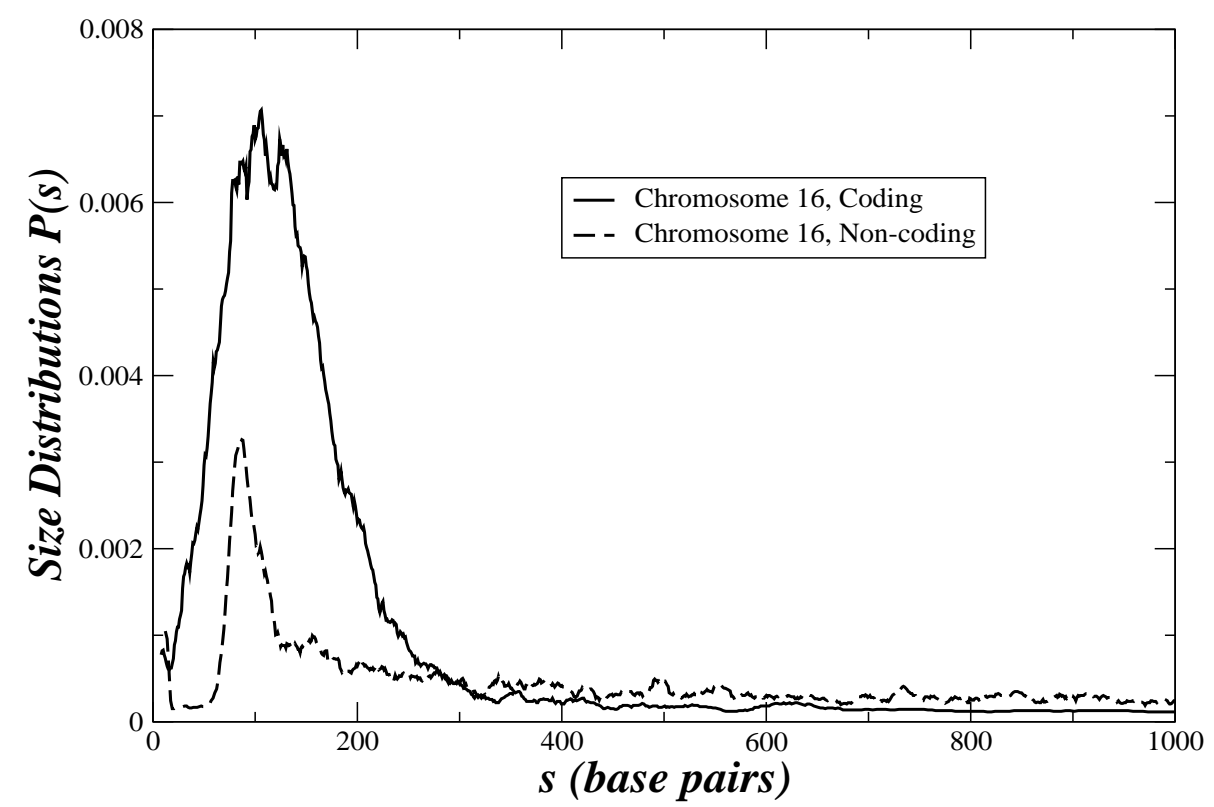

Figure 1: Running average over 15 points of the size distribution of coding and non-coding DNA in chromosome 16. Only first 1000 bps are shown.

non-coding regions reach $10^{8}$ bps. The coding size distributions are rich in small segments of the order of 100-110 bps and then fall fast, while the noncoding ones have a similar maximum in the small scales and fall relatively slower. For comparison we also present the size distribution of chromosome 17, in Fig. 2, in double logarithmic scale where the entire $s$-range is shown.

Comparing Figs. 1 and 2 we note that the size distributions of noncoding DNA, has a complex form but we may clearly distinguish two regions: one region at the short scales which is bell-shaped and which describes the introns (non-coding regions within genes) and one region at the larger scales which contains long tail and which describes the non-coding intergenic regions. It is thus natural, at the phenomenological level, to use the dynamics of Eq. 5 for the description of the complex shape of the size distribution of non-coding DNA hoping to capture these two trends, the introns and the intergenic regions.

In the current study we use non-extensive statistics to study the size distributions of coding and non-coding sequences in the human genome which is now near completion. We have selected to study 4 of the most complete human chromosomes including chromosome 19 which contains the highest 


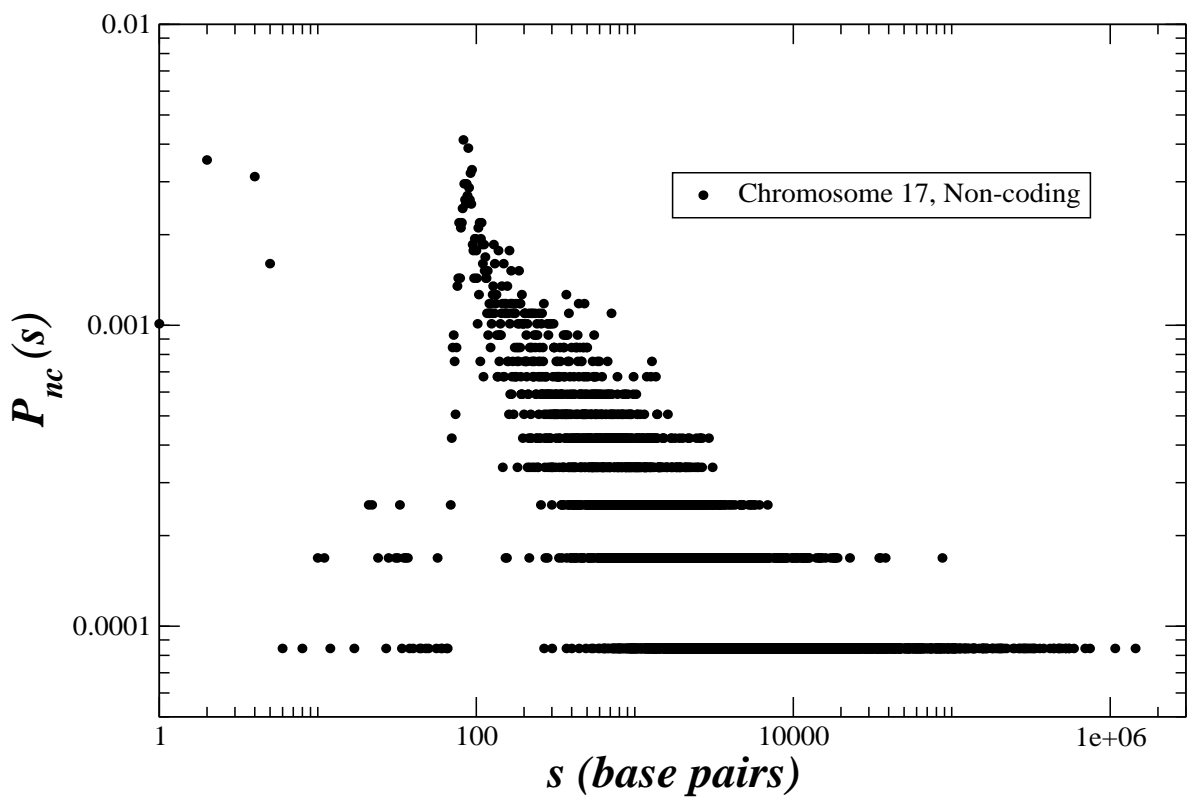

Figure 2: The size distribution of non-coding DNA in chromosome 17 in a double logarithmic scale (all data).

percentage of coding. In the next section we concentrate on the primary structure of the human genome and we give details on the particular data we use. In sections 3 and 4 we present the analysis of the size distribution of coding and non-coding DNA, respectively. We conclude by summarising our main results and discussing some open problems.

\section{The Human Genome}

Although officially the human genome project is announced to be near completion, in the international EMBL and GenBank genomic data bases the sequence data deposited varies from $98.91 \%$ for chromosome 17 to $43.1 \%$ for chromosome Y. The unknown base pairs are usually denoted by the letter $N=$ (unknown base pair) and they are either isolated or appear in clusters. The meaning of $N$ is not unique. It might denote a base pair which resists to sequencing methods completely or partially. Resisting partially means that partial information on the base is known, for example being a Purine or a Pyrimidine. Another case is that the various laboratories which verify the sequencing may not agree on this base pair. 
In the current project we analyse the complete primary structures of human chromosomes $6,16,17$ for which the $N$ percentage is the smallest and also chromosome 19, which contains the highest percentage of coding DNA, 3.8\%. The sequenced percentage presented in the data bases and the coding percentage of these are shown in Table 1. After downloading the chromosomes we isolate the coding and non-coding segments and calculate their respective size distributions for each of them. Representative plot is shown in Fig. 2. Due to the heavy fluctuations in the data we prefer to work with the cumulative distributions $\tilde{P}(s)$ defined as:

$$
\tilde{P}(s)=\int_{s}^{\infty} P(l) d l
$$

where $P(l)$ is the usual distribution of coding or non-coding regions of size $l$. In general, due to summation the cumulative distributions have better statistical properties than the usual distribution functions while they keep the main data trends. Notice that, if the distribution $P(l)$ has the exponential (short range) form its cumulative $\tilde{P}(s)$ will also have the exponential form. If the distribution function has a power law form of the type:

$$
P(l) \sim l^{-1-\mu}
$$

then the cumulative distribution will have a power law form with exponent $-\mu$,

$$
\tilde{P}(s)=\int_{s}^{\infty} l^{-1-\mu} d l=s^{-\mu}, \quad 0 \leq \mu \leq 2 .
$$

Cumulative diagrams of the four coding and non-coding cumulative size distributions are shown in Figs. 3 and 4. respectively. The non-extensive analysis of these distributions follows in the next two sections.

\section{Coding DNA}

As we have already seen in Fig. 1 the coding size distributions have a bell-shape and their tails in the large scales fall relatively fast. To give a quantitative account for the decay of the tails of the distributions we plot the cumulative size distributions in Fig. 3] (solid lines).

To describe the shape of the four curves we use the phenomenological non-extensive description of Eq. 5] and the corresponding curves are also shown in the same figures (dashed lines). The theoretical lines approximate well the data. The exponents $q$ which describe the tails of the distributions 

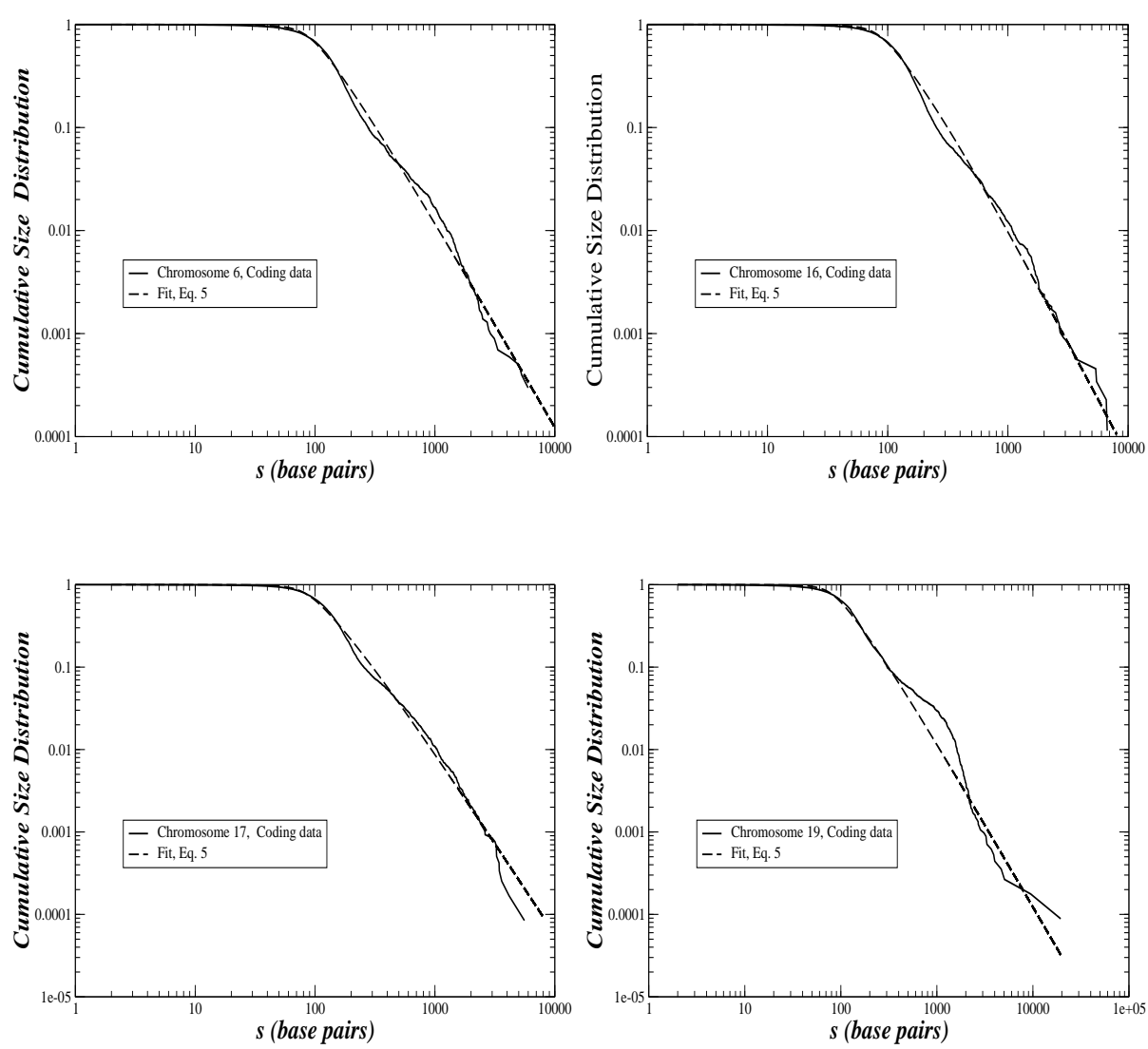

Figure 3: The cumulative size distributions of coding DNA in chromosomes 6, 16, 17 and 19 (solid lines) and the non-linear fits using Eq. [5] (dashed lines).

vary between $1.45<q<1.50$ for the four chromosomes and their specific values are given in Table 1. The non-extensive exponent $q$ corresponds to power law tails of the form Eq. 7 with exponent $\mu$ given by

$$
\mu=-1 /(1-q) .
$$

Thus the tails of the coding size distributions present short range correlations, since $\mu \geq 2$. The exponent $r$ which expresses the small scale characteristics, takes values between $5.2<r<5.6$ for these chromosomes. Similar results have also been observed for the other human chromosomes. The similarity of the two exponents in the four chromosomes indicate that the same (or similar) dynamical processes have created the coding parts of all 


\begin{tabular}{|c|c|c|c|c|c|c|c|}
\hline $\begin{array}{c}\text { Chromo- } \\
\text { some }\end{array}$ & $\begin{array}{c}\text { Sequenced } \\
\%\end{array}$ & $\begin{array}{c}\text { Coding } \\
\%\end{array}$ & $q$ & $r$ & $\kappa_{q}$ & $\lambda_{r}$ & $\begin{array}{c}\mu= \\
1 /(\mathrm{q}-1)\end{array}$ \\
\hline 6 & 97.86 & 1.03657 & 1.50 & 5.2 & 0.018 & 0.00012 & 2.00 \\
\hline 16 & 88.81 & 1.67416 & 1.45 & 5.7 & 0.017 & 0.00009 & 2.22 \\
\hline 17 & 98.91 & 2.48184 & 1.45 & 5.4 & 0.018 & 0.00010 & 2.22 \\
\hline 19 & 87.43 & 3.39768 & 1.50 & 5.6 & 0.018 & 0.00012 & 2.20 \\
\hline
\end{tabular}

Table 1: Non-extensive exponents and parameters describing the coding size distributions.

chromosomes during evolution. This dynamics must be of conservative type in short time scales, since coding DNA changes very slowly (behaves as an almost-closed system) and this is consistent with short range correlations 13. As the human genome annotation advances, we expect that the exponents $r$ and $q$ may be modified and/or other exponents may be needed for a more complete statistical non-extensive description.

\section{Non-Coding DNA}

The cumulative size distributions of the non-coding DNA in the four chromosomes are shown in Fig. 4 (solid lines). We observe that the four distributions have as common characteristic a long tail which can be expressed in the form of a pure power law [12. In the smaller scales the decay is characterised by a different exponent which is very similar for the four distributions.

To describe the shape of the four curves we use the phenomenological non-extensive description of. Eq. 5 and the corresponding curves are shown in the same figures (dashed lines). The theoretical lines are very faithful approximations to the data. The exponents $q$ which describe the long tails of the distributions are very close for the four chromosomes and their corresponding values are given in Table 2, Their values vary between $1.50<q<1.72$. The non-extensive exponent $q$ corresponds to a power law of the form Eq. 7 with exponent $\mu$ being within the bounds $0 \leq \mu \leq 2$, which indicates clear long range correlations. In the case of chromosome 19, which (up to now) contains the highest coding percentage amongst all human chromosomes, the value of $\mu$ calculated through Eq. 9 is equal to 2, which is border line case between short and long range correlations. On the other hand, the exponent $r$ which expresses the small scale characteristics, 
takes values between $2.4<r<3.2$ for these chromosomes. Similar results
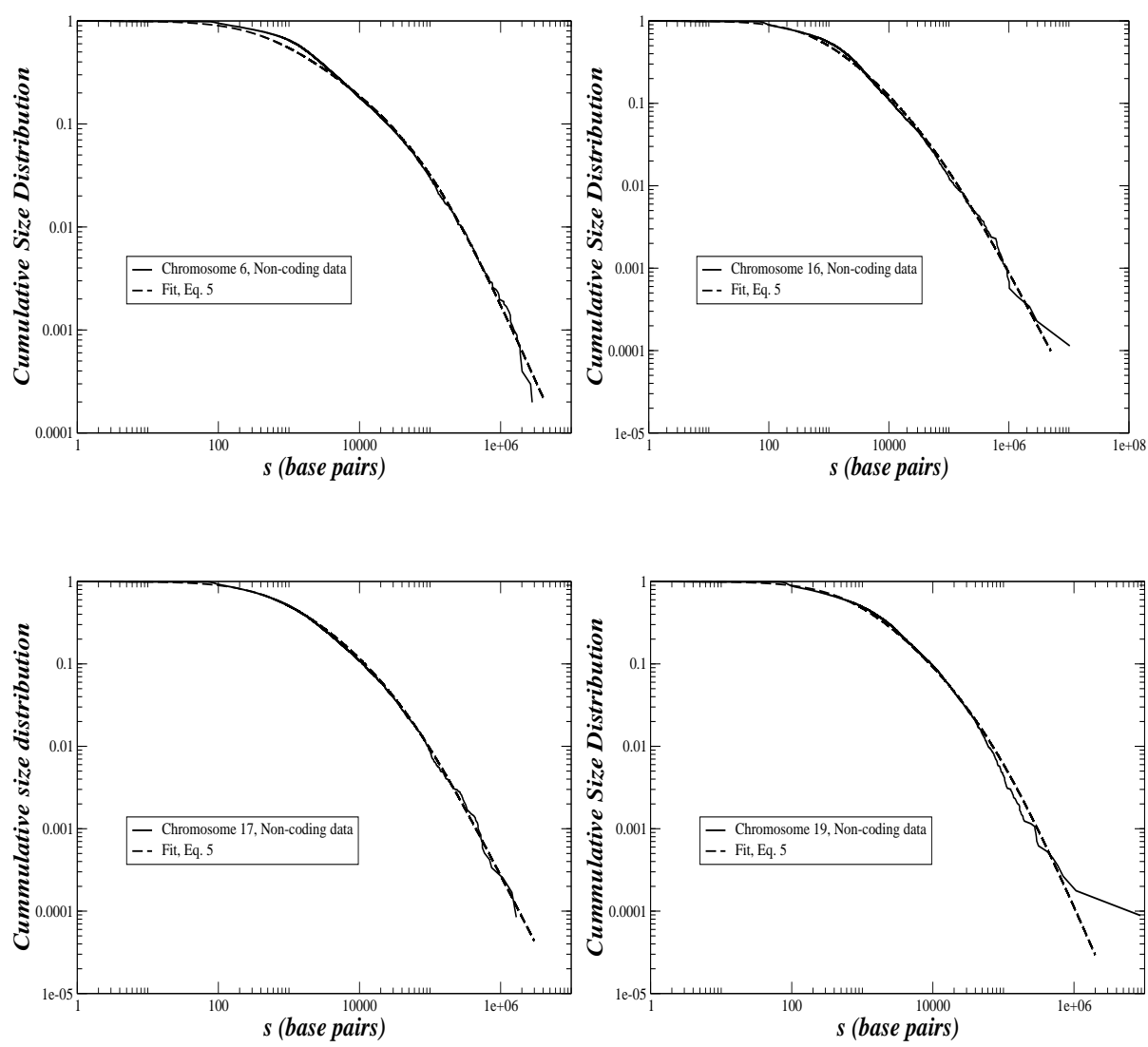

Figure 4: The cumulative size distributions of non-coding DNA in chromosomes 6, 16, 17 and 19 (solid lines) and the non-linear fits using Eq. 5 (dashed lines).

The different small and large scale behaviors observed in the size distribution of the non-coding indicates that different dynamical mechanisms are involved in the formation of small non-coding segments (which are usually found as introns in the genes) and in the large non-coding areas, or intergenic regions which are found between genes and between families of genes. The intergenic regions are extended non-coding regions which can support extensive (massive) influx and outflux of genomic material. Thus the ensemble of intergenic regions acts as an open system which supports exchange with the environment. In open systems, out of equilibrium, power 


\begin{tabular}{|c|c|c|c|c|c|}
\hline $\begin{array}{c}\text { Chromo- } \\
\text { some }\end{array}$ & $q$ & $r$ & $\kappa_{q}$ & $\lambda_{r}$ & $\begin{array}{c}\mu= \\
1 /(q-1)\end{array}$ \\
\hline 6 & 1.65 & 3.2 & 0.00009 & 0.00120 & 1.54 \\
\hline 16 & 1.72 & 2.7 & 0.00021 & 0.00124 & 1.39 \\
\hline 17 & 1.59 & 2.7 & 0.00021 & 0.00118 & 1.69 \\
\hline 19 & 1.50 & 2.4 & 0.00018 & 0.00124 & 2.00 \\
\hline
\end{tabular}

Table 2: Non-extensive exponents and parameters describing the noncoding size distributions.

laws and long range correlations which may be regarded as expression of non-extensive or edge of chaos dynamics, naturally emerge. Open aggregating systems, with influx mechanisms similar to the ones involved in genomic evolution and which lead to long range correlations are presented in reference [13. On the other hand, the non-coding segments found within genes, called also introns are less supportive to external influences because often they include functional strings. Thus they behave more like closed systems and thus the dynamics must be very different, which is also expressed by the difference in the exponents $q$ and $r$.

\section{Conclusions}

We have studied the size distribution of all known coding and non-coding sequences in human chromosomes $6,16,17$ and 19. The first three were selected as representatives of the most completely sequenced chromosomes while chromosome 19 has the highest, up to date, coding percentage. We have found that the decay of the non-coding size distributions is consistent with non-extensive dynamics as expressed by non-linear Eq. 5. Moreover, we have shown that the non-coding presents two distinct regions, one large scale region, related to the intergenic non-coding DNA which presents a decaying exponent $1.5 \leq q \leq 1.72$, and a second short scale region related to the introns (non-coding DNA within genes) which presents a decaying exponent $r>2.4$. On the contrary, short range correlations have been observed in the tails of the coding distributions with non-extensive exponents $1.45 \leq q \leq$ 1.50. This is consistent with earlier observed long (short) range correlations in the non-coding (coding) size distributions of higher eucaryotes. All other human chromosomes demonstrate similar characteristics. 
A more detailed analysis could involve the use of more terms with different exponents in Eq. [5] in order to capture more details such as the dynamical exponent which govern non-coding distances between families of homologous genes (they may be governed by one of the current exponents, $q$ or $r$, or by a third one).

It is true that today the human chromosomes may be close to full sequencing but their complete annotation will take much longer. This means that there are still coding sequences which are not discovered within the genome. Thus we expect that with the advancement of DNA annotation, which is the next major step in genomics after sequencing, we will be able to give more precise, final values to the exponents $q$ and $r$ for the human genome. Also the study in parallel of the genomes of other organisms, as they become sequenced and annotated, will allow for a comparative analysis of genomic data between different classes of organisms.

\section{Acknowledgments}

The authors would like to thank Prof. C. Tsallis for suggesting this approach and Prof. K. Trougkos for helpful discussions.

\section{References}

[1] C.-K. Peng, S. V. Buldyrev, A. L. Goldberger, S. Havlin, F. Sciortino, M. Simons and H. E. Stanley, Nature 356, 168 (1992).

[2] W. Li and K. Kaneko, Europhys. Lett. 17, 655 (1992).

[3] R. F. Voss, Phys. Rev. Lett., 68, 3805 (1992).

[4] R. N. Mantegna, S. V. Buldyrev, A. L. Goldberger, S. Havlin, C. -K. Peng, M. Simons and H. E. Stanley, Phys. Rev. E, 52, 2939 (1995).

[5] A. Provata and Y. Almirantis, Physica A, 247, 482 (1997).

[6] A. Arneodo, E. Bacry, P. V. Graves and J. F. Muzy, Phys. Rev. Lett., 74, 3293 (1995); A. Arneodo, Y. d' Aubenton-Carafa, E. Bacry, P. V. Graves, J. F. Muzy and C. Thermes, Physica D, 96, 291 (1996).

[7] R.N. Mantegna, S.V. Buldyrev, A.L. Goldberger, S. Havlin, C.-K. Peng, M. Simons and H.E. Stanley, Phys. Rev. Letts, 73, 3169 (1994); A. Czirók, R.N. Mantegna, S. Havlin and H.E. Stanley, Physical Review $E, \mathbf{5 2}, 446$ (1995). 
[8] A. A. Tsonis, J. B. Elsner and P. A. Tsonis, J. Theor. Biol., 151, 323 (1991).

[9] S. Karlin and V. Brendel, Science, 259, 677 (1993).

[10] H. Herzel, E. N. Trifonov, O. Weiss and I. Grosse, Physica A, 249, 449 (1998).

[11] S.V. Buldyrev, A.L. Goldberger, S. Havlin, C.-K. Peng, M. Simons and H.E. Stanley, Phys. Rev E, 47, 4514 (1993).

[12] Y. Almirantis and A. Provata, J. Stat. Phys., 97233 (1999).

[13] A. Provata and Y. Almirantis, J. Stat. Phys., 106, 23 (2002).

[14] P. Katsaloulis, T. Theoharis and A. Provata, Physica A, 316, 380 (2002).

[15] S. Abe and Y. Okamoto, eds., Nonextensive Statistical Mechanics and its Applications, Series Lecture Notes in Physics (Springer-Verlag, Berlin, 2001); G. Kaniadakis, M. Lissia and A. Rapisarda, eds., Non Extensive Statistical Mechanics and Physical Applications, Physica A 305, No 1/2 (Elsevier, Amsterdam, 2002).

[16] I. Bediaga, E.M.F. Curado and J. Miranda, Physica A 286, 156 (2000).

[17] C. Beck, Phys. Rev. Lett. 87, 180601 (2001); N. Arimitsu and T. Arimitsu, Europhys. Lett. 60, 60 (2002); M. Peyrard and I. Daumont, Europhys. Lett. bf 59, 834 (2002).

[18] A. Upadhyaya, J.-P. Rieu, J.A. Glazier and Y. Sawada, Physica A 293, 549 (2001).

[19] P. A. Alemany and D. H. Zanette, Phys. Rev. E 49, R956 (1994); C. Tsallis, S. V. F. Levy, A. M. C. Souza and R. Maynard, Phys. Rev. Lett. 75, 3589 (1995); A.R. Plastino and A. Plastino, Physica A 222, 347 (1995).

[20] M. L. Lyra and C. Tsallis, Phys. Rev. Lett. 80, 53 (1998); E.P. Borges, C. Tsallis, G.F.J. Ananos and P. M. C. Oliveira, Phys. Rev. Lett. 89, 254103 (2002); Y.S. Weinstein, S. Lloyd and C. Tsallis, Phys. Rev. Lett. 89, 214101 (2002).

[21] V. Latora, A. Rapisarda and C. Tsallis, Phys. Rev. E 64, 056134 (2001). 
[22] G. A. Tsekouras, A. Provata and C. Tsallis, Phys. Rev. E 69, 016120 (2004).

[23] C. Tsallis, J. Stat. Phys., 52, 479, (1988); E. M. F. Curado and C. Tsallis, J. Phys. A 24 L69 (1991); C. Tsallis, R.S. Mendes and A.R. Plastino, Physica A 261, 534 (1998).

[24] C. Tsallis, G. Bemski and R. S. Mendes Phys. Letts. A 257, 93 (1999). 